Bulletin of the Section of Logic

Volume 48/2 (2019), pp. 137-158

http://dx.doi.org/10.18778/0138-0680.48.2.04

Paolo Maffezioli and Eugenio Orlandelli

\title{
FULL CUT ELIMINATION AND INTERPOLATION FOR INTUITIONISTIC LOGIC WITH EXISTENCE PREDICATE $^{1}$
}

\begin{abstract}
In previous work by Baaz and Iemhoff, a Gentzen calculus for intuitionistic logic with existence predicate is presented that satisfies partial cut elimination and Craig's interpolation property; it is also conjectured that interpolation fails for the implication-free fragment. In this paper an equivalent calculus is introduced that satisfies full cut elimination and allows a direct proof of interpolation via Maehara's lemma. In this way, it is possible to obtain much simpler interpolants and to better understand and (partly) overcome the failure of interpolation for the implication-free fragment.
\end{abstract}

Keywords: intuitionistic logic, existence predicate, sequent calculi, cut elimination, interpolation, Maehara's lemma.

\section{Introduction}

In [9] Scott introduced intuitionistic logic with existence predicate (ILE) to make explicit the existential assumptions in an intuitionistic theory, that is to indicate which objects exist. In ILE the language of first-order intuitionistic logic is extended with an existence predicate $\mathcal{E}$ and $\mathcal{E} t$ is interpreted as saying that (the object denoted by) $t$ exists. In [9] ILE is presented as an Hilbert system extending the standard axiomatization of intuitionistic propositional logic with new rules for quantifiers and axioms for $\mathcal{E}$. In [1, 2] Baaz and Iemhoff introduced Gentzen systems equivalent to

\footnotetext{
${ }^{1}$ Thanks to an anonymous referee for many helpful comments.
} 
Scott's axiomatization and showed that they satisfy partial cut elimination as well as Craig's interpolation property. The proof of the latter, however, is indirect in the sense that interpolation is proved not for the original calculus, but in an equivalent one where structural rules are not admissible. Moreover, it is conjectured that interpolation fails for the implication-free fragment.

The aim of this paper is to improve on $[1,2]$. We introduce an alternative Gentzen calculus for ILE that satisfies full cut elimination and in which Craig's interpolation property can be proved via Maehara's lemma using exclusively the rules of the calculus. The advantage is that our proof is direct and delivers much simpler interpolants. This helps to improve on the conjecture of Baaz and Iemhoff $[1, \S 5.1]$ that their calculi do not interpolate for the fragment of the language without implication (nor $\perp$ ). Specifically, we prove (Proposition 12) that although the interpolants for the implication-free fragment may contain implications, the antecedent of such implications is always an existence atom - and not an arbitrary formula as in [1]. Moreover, we are able to calculate a precise upper bound to the number of such implications. Finally, we prove (Proposition 13) that under an arguably plausible assumption our calculi interpolate for the fragment of the language without implication (nor $\perp$ ).

The paper also improves on other works in the area of interpolation for first-order theories, especially [4] where it is shown how to extend interpolation to a class of first-order theories, called singular geometric, where individual constants do not occur. Since in ILE, constants do occur in existential axioms $\mathcal{E} t$, it is clear that the proof of interpolation presented here indicates a way to generalize the results of [4].

\section{The calculi LJE and $\operatorname{LJE}\left(\Sigma_{\mathcal{L}}\right)$}

To make the paper self-contained we recall basic definitions and results from $[1,2]$. Let $\mathcal{L}^{\prime}$ be a first-order language without identity and let LJE be a Gentzen calculus consisting of the initial sequents and rules given in Table 1.

In the rules $R \forall$ and $L \exists$, the variable $y$ is eigenvariable, i.e. it does not occur free in the conclusion of the rule. ${ }^{2}$ Moreover, let $\Sigma_{\mathcal{L}}$ be the set of all

\footnotetext{
${ }^{2}$ In $[1,2]$ the substitution occurs in the conclusion of the rules $R \forall$ and $L \exists$ instead of in their premises, but this difference is immaterial.
} 
Table 1. The calculus LJE

$$
\begin{array}{cc}
\hline P, \Gamma \Rightarrow P & \overline{\perp, \Gamma \Rightarrow C}^{L \perp} \\
\frac{A, B, \Gamma \Rightarrow C}{A \wedge B, \Gamma \Rightarrow C} L \wedge & \frac{\Gamma \Rightarrow A \quad \Gamma \Rightarrow B}{\Gamma \Rightarrow A \wedge B} R \wedge \\
\frac{A, \Gamma \Rightarrow C \quad B, \Gamma \Rightarrow C}{A \vee B, \Gamma \Rightarrow C} L \vee & \frac{\Gamma \Rightarrow A}{\Gamma \Rightarrow A \vee B}{ }^{R \vee}{ }_{1} \frac{\Gamma \Rightarrow B}{\Gamma \Rightarrow A \vee B} R \vee_{2} \\
\frac{A \rightarrow B, \Gamma \Rightarrow A \quad B, \Gamma \Rightarrow C}{A \rightarrow B, \Gamma \Rightarrow C} L \rightarrow & \frac{A, \Gamma \Rightarrow B}{\Gamma \Rightarrow A \rightarrow B} R \rightarrow \\
\frac{\forall x A, \Gamma \Rightarrow \mathcal{E} t \quad A\left[\begin{array}{l}
t \\
x
\end{array}\right], \forall x A, \Gamma \Rightarrow C}{\forall x A, \Gamma \Rightarrow C} L \forall^{*} & \frac{\mathcal{E} y, \Gamma \Rightarrow A\left[\begin{array}{l}
y \\
x
\end{array}\right]}{\Gamma \Rightarrow \forall x A} R \forall \\
\frac{\mathcal{E} y, A\left[\begin{array}{l}
y \\
x
\end{array}\right], \Gamma \Rightarrow C}{\exists x A, \Gamma \Rightarrow C} L \exists & \frac{\Gamma \Rightarrow \mathcal{E} t \quad \Gamma \Rightarrow A\left[\begin{array}{l}
t \\
x
\end{array}\right]}{\Gamma \Rightarrow \exists \exists^{*}}
\end{array}
$$

sequents $\Gamma \Rightarrow \mathcal{E}$, where $t$ is a term of a language $\mathcal{L} \subseteq \mathcal{L}^{\prime}$ which contains no variable and at least one constant (hence $t$ is a constant). Since $\mathcal{L}$ contains at least one constant, $\Sigma_{\mathcal{L}}$ is not empty; and since $\mathcal{L}$ contains no variable, all sequents in $\Sigma_{\mathcal{L}}$ are closed. Consider now the calculus $\operatorname{LJE}\left(\Sigma_{\mathcal{L}}\right)$ obtained from LJE by adding all the sequents in the language $\mathcal{L}^{\prime}$ that are LJE-derivable from $\Sigma_{\mathcal{L}}$. In other words, $\operatorname{LJE}\left(\Sigma_{\mathcal{L}}\right)$ is obtained from LJE by adding "axiomatic sequents" $\Gamma \Rightarrow \mathcal{E}$ t.

In [2] it is shown that $\operatorname{LJE}$ and $\operatorname{LJE}\left(\Sigma_{\mathcal{L}}\right)$ are equivalent to the standard axiomatizations IQCE and IQCE ${ }^{+}$of ILE due to Scott [9] and Beeson [3], respectively. In [2] it is also shown that in $\operatorname{LJE}$ and $\operatorname{LJE}\left(\Sigma_{\mathcal{L}}\right)$ weakening and contraction

$$
\frac{\Gamma \Rightarrow C}{A, \Gamma \Rightarrow C} \text { Wkn } \quad \frac{A, A, \Gamma \Rightarrow C}{A, \Gamma \Rightarrow C} C t r
$$

are height-preserving admissible (Lemma 4.3 and 4.4). However, the presence of axiomatic sequents in $\operatorname{LJE}\left(\Sigma_{\mathcal{L}}\right)$ impairs cut elimination: although LJE is fully cut-free, $\operatorname{LJE}\left(\Sigma_{\mathcal{L}}\right)$ only allows a partial cut elimination. Specif- 
ically, the cut rule with $\mathcal{E}$-formulas principal (with $t$ term in $\mathcal{L}$ )

$$
\frac{\Gamma \Rightarrow \mathcal{E} t \quad \mathcal{E} t, \Delta \Rightarrow C}{\Gamma, \Delta \Rightarrow C} \text { Cut }
$$

is not eliminable (Theorem 4.6).

Interpolation in $\operatorname{LJE}\left(\Sigma_{\mathcal{L}}\right)$ is investigated in [1] where it is shown LJE and $\operatorname{LJE}\left(\Sigma_{\mathcal{L}}\right)$ have Craig's interpolation property (Corollary 2). The proof is indirect in the sense that interpolation is not proved for $\operatorname{LJE}\left(\Sigma_{\mathcal{L}}\right)$, but for an equivalent system where weakening and contraction are not admissible and cuts on axiomatic sequents are replaced by instances of weakening (Theorem 4). Towards the end of [1] it is conjectured that interpolation fails for the implication-free fragment of $\operatorname{LJE}$ and $\operatorname{LJE}\left(\Sigma_{\mathcal{L}}\right)$ because the proof of interpolation in the case of the rule $L \forall$ only deliver implicative interpolants, i.e. formulas of the form $A \rightarrow B$.

\section{The cut-free calculi G3ie and G3ie ${ }^{\mathcal{T}}$}

To overcome the limitation of partial cut elimination, we consider a calculus equivalent to $\operatorname{LJE}\left(\Sigma_{\mathcal{L}}\right)$ where the rules for quantifiers are aptly modified and each existential sequent $\Gamma \Rightarrow \mathcal{E} t$ in $\Sigma_{\mathcal{L}}$ is replaced by an inference rule. The modification of the quantifier rules has been largely inspired by the modal rules of labelled sequent calculi of [7] and consists into replacing $L \forall^{*}$ and $R \exists^{*}$ of $\operatorname{LJE}\left(\Sigma_{\mathcal{L}}\right)$ by the following rules:

$$
\frac{A\left[\begin{array}{l}
t \\
x
\end{array}\right], \forall x A, \mathcal{E} t, \Gamma \Rightarrow C}{\forall x A, \mathcal{E} t, \Gamma \Rightarrow C} L \forall \quad \frac{\mathcal{E} t, \Gamma \Rightarrow A\left[\begin{array}{l}
t \\
x
\end{array}\right]}{\mathcal{E} t, \Gamma \Rightarrow \exists x A} R \exists
$$

Let G3ie be the result of replacing $L \forall^{*}$ and $R \exists^{*}$ of $\operatorname{LJE}\left(\Sigma_{\mathcal{L}}\right)$ with $L \forall$ and $R \exists$, respectively. The key feature of $L \forall$ and $R \exists$ in G3ie is that existential atoms may be active (principal) only in the left-hand side of the sequent arrow $\Rightarrow$.

Next, instead of axiomatic sequents $\Gamma \Rightarrow \mathcal{E} t$, we consider (extensions of G3ie with) existential rules $E x(t)$ of the form

$$
\frac{\mathcal{E} t, \Gamma \Rightarrow C}{\Gamma \Rightarrow C} \operatorname{Ex}(t)
$$

where $t$ is a term of $\mathcal{L}$. We agree that if $\mathcal{T}=\left\{t: \Gamma \Rightarrow \mathcal{E} t \in \Sigma_{\mathcal{L}}\right\}$, then $\mathrm{G} \mathrm{e}^{\mathcal{T}}$ is the extension of G3ie with a rule $\operatorname{Ex}(t)$ for each $t \in \mathcal{T}$. 
Now we show that G3ie ${ }^{\mathcal{T}}$ (as well as G3ie) satisfies full cut elimination. As usual, we shall assume that derivations satisfy the pure-variable convention: in a derivation no variable occurs both free and bound and the eigenvariables are pairwise disjoint. Next, we begin with some preparatory lemmas. First, height-preserving admissibility of substitution in G3ie ${ }^{\mathcal{T}}$ (we shall omit to specify $\mathrm{G}^{\mathrm{T}} \mathrm{e}^{\mathcal{T}}$, unless it is necessary).

Lemma 1 (substitution). If $\vdash^{h} \Gamma \Rightarrow C$ and $t$ is free for $x$ in $\Gamma, C$ then $\vdash^{h} \Gamma\left[\begin{array}{l}t \\ x\end{array}\right] \Rightarrow C\left[\begin{array}{l}t \\ x\end{array}\right]$.

Proof: By induction on height $h$ of the derivation of $\Gamma \Rightarrow C$. If $h=0$ or $h=n+1$ and the last rule instance $R$ is by a propositional rule, see [8, Theorem 4.1.2]. If ( $h=n+1$ and) $R$ is $L \forall$ and $x \equiv y$ then the claim holds since the substitution $\left[\begin{array}{c}t \\ x\end{array}\right]$ is vacuous. Otherwise, if $x \not \equiv y$ then we apply IH on the premise of $L \forall$ and then $L \forall$ again. The case of $R \exists$ is similar. If $R$ is $R \forall$ with conclusion $\Gamma \Rightarrow \forall y A$, then we take the premise $\mathcal{E} z, \Gamma \Rightarrow A\left[\begin{array}{c}z \\ y\end{array}\right]$ (with $z$ eigenvariable) and we apply IH so as to replace $z$ with a new variable $u$ and obtain $\vdash^{n} \mathcal{E} u, \Gamma \Rightarrow A\left[\begin{array}{l}u \\ y\end{array}\right]$. By IH again and $R \forall$ we conclude $\Gamma\left[\begin{array}{l}t \\ x\end{array}\right] \Rightarrow(\forall y A)\left[\begin{array}{l}t \\ x\end{array}\right]$. The case of $L \exists$ is similar.

Next is height-preserving admissibility of weakening. Lemma 2 (Weakening). If $\vdash^{h} \Gamma \Rightarrow C$ then $\vdash^{h} A, \Gamma \Rightarrow C$.

Proof: By induction on $h$. If $h=0$ or $h=n+1$ and the last rule instance $R$ is by a propositional rule, see [8, Theorem 4.2.2]. If $(h=n+1$ and $)$ $R$ is a quantifier rule without variable condition, then the claim holds by IH and $R$. If $R$ is a quantifier rule with eigenvariable then use Lemma 1 , $\mathrm{IH}$ and $R$. Finally, if $R$ is $E x(t)$, then its premise is $\mathcal{E} t, \Gamma \Rightarrow C$ and the conclusion $A, \Gamma \Rightarrow C$ is obtained by applying IH on the premise of $E x(t)$ and then $\operatorname{Ex}(t)$ (since $t$ is a constant, we know $\mathcal{E} t$ is not affected by the substitution).

To prove that contraction is height-preserving admissible, we need height-preserving invertibility of some rules.

Lemma 3 (Inversion). All rules, except $R \vee, L \rightarrow$ and $R \exists$, are heightpreserving invertible. However, $L \rightarrow$ is height-preserving invertible with respect to its right premise.

Proof: For height-preserving invertibility of the propositional rules, see [8, Theorem 2.3.5]. The height-preserving invertibility of $L \forall$ and $E x(t)$ follows by height-preserving admissibility of weakening (Lemma 2), whereas 
the case of $L \exists$ is as in [2, Lemma 4.4] and the case of $R \forall$ is similar and hence left to the reader.

Now we can prove height-preserving admissibility of contraction. Lemma 4 (Contraction). If $\vdash^{h} A, A, \Gamma \Rightarrow C$ then $\vdash^{h} A, \Gamma \Rightarrow C$.

Proof: By induction on $h$. If $h=0$ or $h=n+1$ and the last rule instance $R$ is by a propositional rule, see [8, Theorem 2.4.1]. If $R$ is a height-preserving invertible quantifier rule, then the claim holds by $\mathrm{IH}$ and Lemma 3. If $R$ is $R \exists$, then $A$ cannot be principal in it and $C \equiv \exists x B$. We need to consider two cases: either $A \equiv \mathcal{E} t$ or $\Gamma \equiv \mathcal{E} t, \Gamma^{\prime}$. In the first case, the premise of $R \exists$ is $\mathcal{E} t, \mathcal{E} t, \Gamma \Rightarrow B\left[\begin{array}{l}t \\ x\end{array}\right]$ and the sequent $\mathcal{E} t, \Gamma \Rightarrow \exists x B$ is obtained by applying IH on $\mathcal{E} t$ and then $R \exists$ again. In the second case, the premise of $R \exists$ is $A, A, \mathcal{E} t, \Gamma^{\prime} \Rightarrow B\left[\begin{array}{c}t \\ x\end{array}\right]$ and the sequent $A, \mathcal{E} t, \Gamma^{\prime} \Rightarrow \exists x B$ is obtained similarly. Finally, if $R$ is $\operatorname{Ex}(t)$, then $A$ cannot be principal in it and the premise of $\operatorname{Ex}(t)$ is $A, A, \mathcal{E} t, \Gamma \Rightarrow C$. Thus, $A, \mathcal{E} t, \Gamma \Rightarrow C$ is obtained by applying IH and then $\operatorname{Ex}(t)$.

We are now ready to prove (full) cut elimination.

Theorem 5 (Cut). If $\vdash \Gamma \Rightarrow A$ and $\vdash A, \Delta \Rightarrow C$ then $\vdash \Gamma, \Delta \Rightarrow C$.

Proof: The proof is by induction on the weight of the cut formula $A$ with a sub-induction on the sum of heights of derivation of the two premises (cut-height, for short). If at least one the two premises of cut is initial or concluded by $L \perp$, then the proof is the same as in [8, Theorem 2.4.3]. Otherwise, if none of the premises of cut is initial or concluded by $L \perp$, we consider three cases: (i) $A$ is not principal in $\Gamma \Rightarrow A$; (ii) $A$ is principal in $\Gamma \Rightarrow A$ only; (iii) $A$ is principal in $\Gamma \Rightarrow A$ and $A, \Delta \Rightarrow C$.

In case (i), we proceed by cases according to the rule $R$ concluding $\Gamma \Rightarrow A$. Since $A$ is not principal by hypothesis, $\Gamma \Rightarrow A$ can only be concluded by a left rule or $E x(t)$. We consider only the case of (ia) $L \forall$, (ib) $L \exists$ and (ic) $E x(t)$, the rest being the same as in [8, Theorem 2.4.3]. If (ia) $\Gamma \Rightarrow A$ is concluded by $L \forall$, then $\Gamma \equiv \mathcal{E} t, \forall x B, \Gamma^{\prime}$ and we have:

$$
\frac{\frac{B\left[\begin{array}{l}
t \\
x
\end{array}\right], \mathcal{E} t, \forall x B, \Gamma^{\prime} \Rightarrow A}{\mathcal{E} t, \forall x B, \Gamma^{\prime} \Rightarrow A} L \forall \quad A, \Delta \Rightarrow C}{\mathcal{E} t, \forall x B, \Gamma^{\prime}, \Delta \Rightarrow C} C u t
$$

We apply IH on the premise of $L \forall$ and then $L \forall$ as follows. 


$$
\frac{B\left[\begin{array}{c}
t \\
x
\end{array}\right], E t, \forall x B, \Gamma^{\prime} \Rightarrow A \quad A, \Delta \Rightarrow C}{\frac{B\left[\begin{array}{l}
t \\
x
\end{array}\right], \mathcal{E} t, \forall x B, \Gamma^{\prime}, \Delta \Rightarrow C}{\mathcal{E} t, \forall x B, \Gamma^{\prime}, \Delta \Rightarrow C}} \mathrm{IH}
$$

If (ib) then $\Gamma \Rightarrow A$ is concluded by $L \exists$ then $\Gamma$ is $\exists x B, \Gamma^{\prime}$. In this case the procedure is similar to case (a), except that we need first to apply Lemma 1 on the premise $\mathcal{E} y, B\left[\begin{array}{l}y \\ x\end{array}\right], \Gamma^{\prime} \Rightarrow A$ of $L \exists$ so as to replace $y$ with a new variable $z$.

Finally, if (ic) $\Gamma \Rightarrow A$ is concluded by $E x(t)$ cut is "permuted upwards" as above.

In case (ii), we proceed by cases according to the rule $R$ concluding $A, \Delta \Rightarrow C$. We consider here only the cases of the quantifier rules and $E x(t)$. If $R$ is $L \forall$ or $R \exists$ then we reason as in case (ia), whereas if $R$ is $R \forall$ or $L \exists$ the reasoning is similar to (ib). Finally, If $R$ is $E x(t)$ we proceed as in (ic).

We now consider the case (iii). If the cut formula $A$ is propositional, then see [8, Theorem 2.4.3]. If $A \equiv \forall x B$, then $\Delta \equiv \mathcal{E} t, \Delta^{\prime}$ and we have

$$
\frac{\mathcal{E} y, \Gamma \Rightarrow B\left[\begin{array}{l}
y \\
x
\end{array}\right]}{\Gamma \Rightarrow \forall x B \quad \frac{B\left[\begin{array}{l}
t \\
x
\end{array}\right], \forall x B, \mathcal{E} t, \Delta^{\prime} \Rightarrow C}{\forall x B, \mathcal{E} t, \Delta^{\prime} \Rightarrow C}} \mathrm{Cut}
$$

where $y$ is eigenvariable in $R \forall$. First, we apply height-preserving admissibility of substitution (Lemma 1 ) on the premise of $R \forall$ in order to replace $y$ with $t$; thus, we obtain $\vdash^{n-1} \mathcal{E} t, \Gamma \Rightarrow B\left[\begin{array}{l}t \\ x\end{array}\right]$, where $n$ is the derivation height of the conclusion of $R \forall$. Then we apply IH twice and height-preserving admissibility of contraction (Lemma 4) as follows.

$$
\frac{\frac{\mathcal{E} y, \Gamma \Rightarrow B\left[\begin{array}{l}
y \\
x
\end{array}\right]}{\mathcal{E} t, \Gamma \Rightarrow B\left[\begin{array}{l}
t \\
x
\end{array}\right]}\left[\begin{array}{l}
t \\
y
\end{array}\right] \quad \frac{\Gamma \Rightarrow \forall x B \quad B\left[\begin{array}{l}
t \\
x
\end{array}\right], \forall x B, \mathcal{E} t, \Delta^{\prime} \Rightarrow C}{\Gamma, B\left[\begin{array}{l}
t \\
x
\end{array}\right], \mathcal{E} t, \Delta^{\prime} \Rightarrow C}}{\frac{\mathcal{E} t, \Gamma, \Gamma, \mathcal{E} t, \Delta^{\prime} \Rightarrow C}{\Gamma, \mathcal{E} t, \Delta^{\prime} \Rightarrow C}} \mathrm{IH}
$$

The case in which the cut formula $A$ is $\exists x B$ is similar. Notice the if $A \equiv \mathcal{E} t$, then such a formula cannot be both principal of a right rule and a left rule.

Thus, the calculus G3ie ${ }^{\mathcal{T}}$ satisfies full cut elimination. We now need to prove that it is equivalent to $\operatorname{LJE}\left(\Sigma_{\mathcal{L}}\right)$. 
TheOrem 6. $\operatorname{LJE}\left(\Sigma_{\mathcal{L}}\right)$ and $\mathrm{GBie}^{\mathcal{T}}$ are equivalent.

Proof: Any existential sequent $\Gamma \Rightarrow \mathcal{E} t$ in $\operatorname{LJE}\left(\Sigma_{\mathcal{L}}\right)$ is clearly derivable in $\mathrm{G} \mathrm{ie}^{\mathcal{T}}$ by the corresponding existential rule $E x(t)$. On the other hand, if the existential rule $E x(t)$ is in $\mathrm{G} 3 \mathrm{e}^{\mathcal{T}}$, then it is admissible in $\operatorname{LJE}\left(\Sigma_{\mathcal{L}}\right)$ since it can be simulated by a (non-eliminable) cut with the axiomatic sequent $\Rightarrow \mathcal{E} t$. Thus we have only to prove that rule $L \forall(R \exists)$ is equivalent to the rule $L \forall^{*}\left(R \exists^{*}\right)$. The following derivation shows that $L \forall$ is admissible in $\operatorname{LJE}\left(\Sigma_{\mathcal{L}}\right)$ :

$$
\frac{\mathcal{E} t, \forall x A, \Gamma \Rightarrow \mathcal{E} t \quad A\left[\begin{array}{l}
t \\
x
\end{array}\right], \forall x A, \mathcal{E} t, \Gamma \Rightarrow C}{\forall x A, \mathcal{E} t, \Gamma \Rightarrow C} L \forall^{*}
$$

and, by Lemmas 2 and 4 and Theorem 5 , the following one shows that $L \forall^{*}$ is admissible in $\mathrm{G} \mathrm{ie}^{\mathcal{T}}$ :

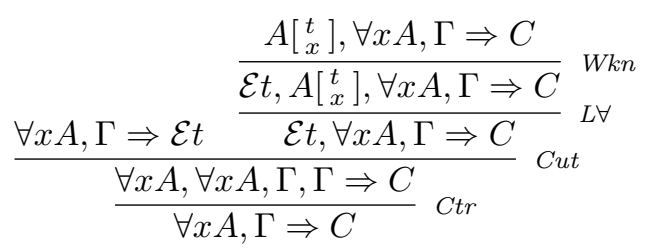

The cases of $R \exists$ in $\operatorname{LJE}\left(\Sigma_{\mathcal{L}}\right)$ and $R \exists^{*}$ in $\mathrm{G}_{3 \mathrm{e}^{\mathcal{T}}}$ are left to the reader.

\section{Interpolation}

We now turn to interpolation. The standard proof of interpolation for Gentzen's calculi LK and LJ rests on cut elimination and a result due to Maehara [5]. We recall from [10] some basic definitions.

Definition 7 (Formula-language). Given a formula $A$ the set $\operatorname{Ter}(A)$ is the set of free variables and individual constants occurring in $A$; the set $\operatorname{Rel}(A)$ is the set of non-logical relational symbols - i.e., all relational symbols except $\mathcal{E}$ - occurring in $A$; and $\operatorname{Lan}(A)$ is the union of $\operatorname{Ter}(A)$ and $\operatorname{Rel}(A)$. These notions are extended to multisets and to sequents as expected.

DEFinition 8 (partition, split-interpolant). A partition of a sequent $\Gamma \Rightarrow$ $C$ is an expression $\Gamma_{1} ; \Gamma_{2} \Rightarrow C$, where $\Gamma=\Gamma_{1}, \Gamma_{2}$ (where $=$ is the multisetidentity). A split-interpolant of a partition $\Gamma_{1} ; \Gamma_{2} \Rightarrow C$ is a formula $I$ such that: 


$$
\begin{aligned}
\text { I } & \vdash \Gamma_{1} \Rightarrow I \\
\text { II } & \vdash I, \Gamma_{2} \Rightarrow C \\
\text { III } & \operatorname{Lan}(I) \subseteq \operatorname{Lan}\left(\Gamma_{1}\right) \cap \operatorname{Lan}\left(\Gamma_{2}, C\right)
\end{aligned}
$$

We use $\Gamma_{1} ; \Gamma_{2} \stackrel{I}{\Rightarrow} C$ to indicate that $I$ is a split-interpolant for $\Gamma_{1} ; \Gamma_{2} \Rightarrow C$.

Moreover, we say that a formula $I$ satisfying conditions (I) and (II) satisfies the derivability conditions for being a split-interpolant for the partition $\Gamma_{1} ; \Gamma_{2} \Rightarrow I$, whereas if $I$ satisfies (III) we say that it satisfies the language condition for being a split-interpolant for the same partition. Given a split sequent $\Gamma_{1} ; \Gamma_{2} \Rightarrow C$, we call $\Gamma_{1}\left(\Gamma_{2}\right)$ its first (second) component. Finally, having assumed that $\mathcal{E} \notin \operatorname{Rel}(A)$ for each formula $A$, we say that $\mathcal{E}$ is a logical predicate.

To prove Maehara's lemma we need first to prove a generalized version of Lemma 1 that allows arbitrary terms (either free variables or individual constants) to be replaced. Thus, we consider a general substitution $\left[\begin{array}{l}t \\ u\end{array}\right]$ of terms for terms and we show its height-preserving admissibility.

Lemma 9 (General substitution). If $\vdash^{n} \Gamma \Rightarrow C$ and $t$ is free for $u$ in $\Gamma, C$ and no instance of the rule Ex(u) has been applied in the derivation of $\Gamma \Rightarrow C$, then $\vdash^{n} \Gamma\left[\begin{array}{l}t \\ u\end{array}\right] \Rightarrow C\left[\begin{array}{l}t \\ u\end{array}\right]$.

Proof: If $u$ is a variable, the claim holds by Lemma 1. Otherwise, let $u$ be an individual constant. We can think of the derivation $\mathcal{D}$ of $\Gamma \Rightarrow C$ as

$$
\frac{\Gamma^{\prime} \Rightarrow C^{\prime}}{\Gamma^{\prime}\left[\begin{array}{l}
u \\
z
\end{array}\right] \Rightarrow C\left[\begin{array}{l}
u \\
z
\end{array}\right]}\left[\begin{array}{l}
u \\
z
\end{array}\right]
$$

where $\Gamma^{\prime} \Rightarrow C^{\prime}$ is like $\Gamma \Rightarrow C$ save that it has a fresh variable $z$ in place of $u$. Note that this is always feasible for purely logical derivations, and it is feasible for derivations involving no instance of rule $E x(u)$. We transform $\mathcal{D}$ into

$$
\frac{\Gamma^{\prime} \Rightarrow C^{\prime}}{\Gamma^{\prime}\left[\begin{array}{l}
t \\
z
\end{array}\right] \Rightarrow C^{\prime}\left[\begin{array}{l}
t \\
z
\end{array}\right]}\left[\begin{array}{l}
t \\
z
\end{array}\right]
$$

where $t$ is free for $z$ since we assumed it is free for $u$ in $\Gamma \Rightarrow C$. We have thus found a derivation $\left(\mathcal{D}\left[\begin{array}{l}t \\ u\end{array}\right]\right)$ of $\Gamma\left[\begin{array}{l}t \\ u\end{array}\right] \Rightarrow C\left[\begin{array}{l}t \\ u\end{array}\right]$ that has the same height as the derivation $\mathcal{D}$ of $\Gamma \Rightarrow C$.

Lemma 10 (Maehara's lemma for G3ie ${ }^{\mathcal{T}}$ ). Every partition $\Gamma_{1} ; \Gamma_{2} \Rightarrow C$ of a derivable sequent $\Gamma \Rightarrow C$ has a split-interpolant. 
Proof: The proof is by induction on the height $h$ of the derivation $\mathcal{D}$. The cases when $h=0$ or $h=n+1$ and the last step in $\mathcal{D}$ is by a propositional rule are identical to the ones for G3i and the reader is referred to [10, §4.4.2] for a sketch of the proof. Hence we have to consider only the rules for the quantifiers and the non-logical rule $E x(t)$. We consider first the rules for the existential quantifier.

Suppose that the last step in $\mathcal{D}$ is by $L \exists$, i.e.

$$
\frac{\mathcal{E} y, A\left[\begin{array}{c}
y \\
x
\end{array}\right], \Gamma \Rightarrow C}{\exists x A, \Gamma \Rightarrow C} L \exists
$$

where $y$ is eigenvariable. We have to consider the following two partitions of the conclusion:

1. $\exists x A, \Gamma_{1} ; \Gamma_{2} \Rightarrow C$

2. $\Gamma_{1} ; \exists x A, \Gamma_{2} \Rightarrow C$

The split-interpolants for these partitions are, respectively,

$$
\frac{A\left[\begin{array}{l}
y \\
x
\end{array}\right], \mathcal{E} y, \Gamma_{1} ; \Gamma_{2} \stackrel{I}{\Rightarrow} C}{\exists x A, \Gamma_{1} ; \Gamma_{2} \stackrel{I}{\Rightarrow} C} \quad \text { and } \quad \frac{\Gamma_{1} ; A\left[\begin{array}{c}
y \\
x
\end{array}\right], \mathcal{E} y, \Gamma_{2} \stackrel{I}{\Rightarrow} C}{\Gamma_{1} ; \exists x A, \Gamma_{2} \stackrel{I}{\Rightarrow} C}
$$

We give the details of the proof only for the first partition since the proof for the other one is almost identical. By induction hypothesis (IH), there is a formula $I$ such that:

(i) $\vdash A\left[\begin{array}{l}y \\ x\end{array}\right], \mathcal{E} y, \Gamma_{1} \Rightarrow I$

(ii) $\vdash I, \Gamma_{2} \Rightarrow C$

(iii) $\operatorname{Lan}(I) \subseteq \operatorname{Lan}\left(A\left[\begin{array}{l}y \\ x\end{array}\right], \mathcal{E} y, \Gamma_{1}\right) \cap \operatorname{Lan}\left(\Gamma_{2}, C\right)$

The following derivations show that $I$ satisfies the derivability conditions for being a split-interpolant of the partition under consideration.

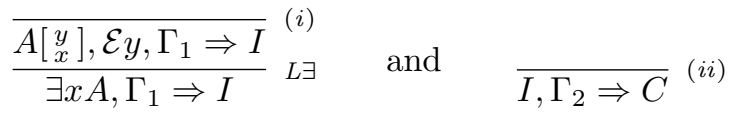


Moreover, since $\mathcal{E} \notin \operatorname{Lan}(I)$ by Definition 7 , to see that (iii) implies that $I$ satisfies the language condition it is enough to notice that $y$ cannot be in $\operatorname{Ter}(I)$ because $y$ is the eigenvariable of this rule instance and, hence, it is not in $\operatorname{Ter}\left(\Gamma_{2}, C\right){ }^{3}$ Now we consider the rule $R \exists$.

Suppose the last step in $\mathcal{D}$ is by $R \exists$ :

$$
\frac{\mathcal{E} t, \Gamma \Rightarrow A\left[\begin{array}{l}
t \\
x
\end{array}\right]}{\mathcal{E} t, \Gamma \Rightarrow \exists x A} R \exists
$$

Once again, we have to consider two partitions:

1. $\Gamma_{1} ; \mathcal{E} t, \Gamma_{2} \Rightarrow \exists x A$

2. $\mathcal{E} t, \Gamma_{1} ; \Gamma_{2} \Rightarrow \exists x A$

For the first partition we have the following split-interpolant:

$$
\frac{\Gamma_{1} ; \mathcal{E} t, \Gamma_{2} \stackrel{I}{\Rightarrow} A\left[\begin{array}{l}
t \\
x
\end{array}\right]}{\Gamma_{1} ; \mathcal{E} t, \Gamma_{2} \stackrel{I}{\Rightarrow} \exists x A}
$$

To see this, notice that by $\mathrm{IH}$ we know that there is a formula $I$ such that:

(i) $\vdash \Gamma_{1} \Rightarrow I$

(ii) $\vdash I, \mathcal{E} t, \Gamma_{2} \Rightarrow A\left[\begin{array}{c}t \\ x\end{array}\right]$

(iii) $\operatorname{Lan}(I) \subseteq \operatorname{Lan}\left(\Gamma_{1}\right) \cap \operatorname{Lan}\left(\mathcal{E} t, \Gamma_{2}, A\left[\begin{array}{l}t \\ x\end{array}\right]\right)$

From (i) and (ii) it immediately follows that $I$ satisfies the derivability condition — we only need to apply $R \exists$ to the sequent in (ii). Moreover, (iii) implies that $I$ satisfies the language condition too, since $\operatorname{Lan}\left(\mathcal{E} t, \Gamma_{2}, \exists x A\right)=$ $\operatorname{Lan}\left(\mathcal{E} t, \Gamma_{2}, A\left[\begin{array}{l}t \\ x\end{array}\right]\right)$ (for $t$ already occurs in both). For the second partition the proof is more complicated. By IH we can assume there is a formula $I$ such that:

(i) $\vdash \mathcal{E} t, \Gamma_{1} \Rightarrow I$

(ii) $\vdash I, \Gamma_{2} \Rightarrow A\left[\begin{array}{l}t \\ x\end{array}\right]$

(iii) $\operatorname{Lan}(I) \subseteq \operatorname{Lan}\left(\mathcal{E} t, \Gamma_{1}\right) \cap \operatorname{Lan}\left(\Gamma_{2}, A\left[\begin{array}{l}t \\ x\end{array}\right]\right)$

3 In [1, p. 11] the split-interpolant of the given partition is identified with $\forall z I\left[\begin{array}{l}z \\ y\end{array}\right]$ where the $\forall z$ can be dropped when $y$ does not occur free in $I$. Our reasoning shows that we are always in this latter case. 
Now we need to consider three mutually incompatible and exhaustive cases:

(a) $t \notin \operatorname{Ter}\left(\Gamma_{2}, \exists x A\right)$

(b) $t \in \operatorname{Ter}\left(\Gamma_{2}, \exists x A\right)$ and $t \in \mathcal{T}$

(c) $t \in \operatorname{Ter}\left(\Gamma_{2}, \exists x A\right)$ and $t \notin \mathcal{T}$

If case (a) holds, the partition has the following split-interpolant:

$$
\frac{\mathcal{E} t, \Gamma_{1} ; \Gamma_{2} \stackrel{I}{\Rightarrow} A\left[\begin{array}{l}
t \\
x
\end{array}\right]}{\mathcal{E} t, \Gamma_{1} ; \Gamma_{2} \stackrel{\exists y I\left[\begin{array}{l}
y \\
t
\end{array}\right]}{\longrightarrow} \exists x A}
$$

Indeed, the following derivations show that $\exists y I\left[\begin{array}{l}y \\ t\end{array}\right]$ satisfies the derivability conditions:

$$
\frac{\overline{\mathcal{E} t, \Gamma_{1} \Rightarrow I}{ }^{(i)}}{\frac{\mathcal{E} y, \Gamma_{1}\left[\begin{array}{l}
y \\
t
\end{array}\right] \Rightarrow\left(I\left[\begin{array}{l}
y \\
t
\end{array}\right]\right)\left[\begin{array}{l}
y \\
y
\end{array}\right]}{\mathcal{E} y, \Gamma_{1}\left[\begin{array}{l}
y \\
t
\end{array}\right] \Rightarrow \exists y I\left[\begin{array}{l}
y \\
t
\end{array}\right]}}\left[\begin{array}{l}
y \\
t
\end{array}\right]\left[\begin{array}{l}
y \\
y
\end{array}\right]
$$

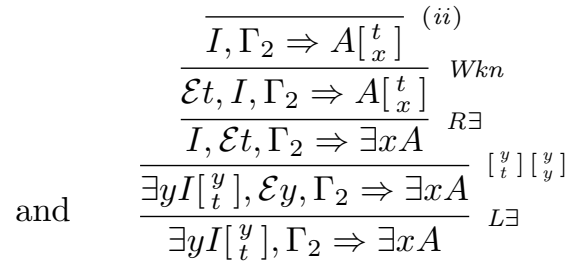

where $y$ is a new variable and in both derivations the inference steps where we have applied substitutions are height-preserving admissible by Lemma 9. In particular, Lemma 9 allows us to apply the substitution $\left[\begin{array}{l}y \\ t\end{array}\right]$ because, thanks to the admissibility of contraction (Lemma 4), we can eliminate any instance of rule $E x(t)$ from the derivation of a sequent where $\mathcal{E} t$ occurs. Moreover, the assumption for case (a), i.e. $t \notin \operatorname{Ter}\left(\Gamma_{2}, \exists x A\right)$, ensures that the substitution $\left[\begin{array}{l}y \\ t\end{array}\right]$ has no effect on $\Gamma_{2}, \exists x A$ in the right derivation. It is also immediate to see that (iii) entails that $\exists y I\left[\begin{array}{l}y \\ t\end{array}\right]$ satisfies the language condition since $t \notin \operatorname{Ter}\left(\exists y I\left[\begin{array}{l}y \\ t\end{array}\right]\right)$. Thus, the split-interpolant is $\exists y I\left[\begin{array}{l}y \\ t\end{array}\right]$ and whenever $t \notin \operatorname{Ter}(I)$, we can drop the vacuous quantification. In case (b), the partition has the following split-interpolant:

$$
\frac{\mathcal{E} t, \Gamma_{1} ; \Gamma_{2} \stackrel{I}{\Rightarrow} A\left[\begin{array}{c}
t \\
x
\end{array}\right]}{\mathcal{E} t, \Gamma_{1} ; \Gamma_{2} \stackrel{I}{\Rightarrow} \exists x A}
$$


Too see this notice that (iii) entails that $I$ satisfies the language condition and the following derivations, where $E x(t)$ is applicable, since $t \in \mathcal{T}$ by assumption for case (b), show it satisfies the derivability conditions as well.

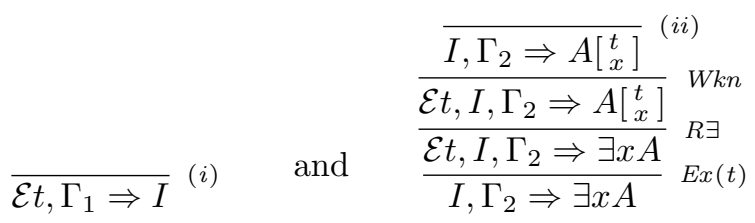

Finally, in case (c) the split-interpolant is:

$$
\frac{\mathcal{E} t, \Gamma_{1} ; \Gamma_{2} \stackrel{I}{\Rightarrow} A\left[\begin{array}{c}
t \\
x
\end{array}\right]}{\mathcal{E} t, \Gamma_{1} ; \Gamma_{2} \stackrel{I \wedge \mathcal{E} t}{\Longrightarrow} \exists x A}
$$

On the one hand, the following derivations show that $I \wedge \mathcal{E} t$ satisfies the derivability conditions:

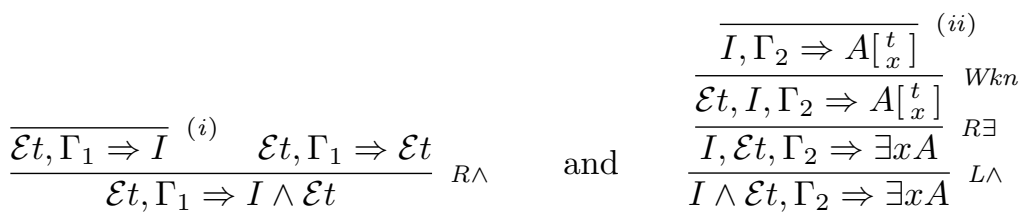

On the other hand, the formula $I \wedge \mathcal{E} t$ satisfies the language condition since both $I$ and $\mathcal{E} t$ satisfy it. Indeed, that $I$ satisfies it follows immediately from (iii). To see that the same holds for $\mathcal{E}$, notice that both $t$ and $\mathcal{E}$ satisfy the language condition in virtue of the assumption for case (c) and the fact that $\mathcal{E}$ is a logical predicate, respectively. ${ }^{4}$ This completes the proof for the rules of the existential quantifier. Next we move to the rules for the universal quantifier.

${ }^{4}$ If, instead, $\mathcal{E}$ weren't a logical predicate, interpolation would fail for ILE altogether: for $P t \wedge \mathcal{E} t \rightarrow \exists x P x$ is a theorem whose interpolant is $P t \wedge \mathcal{E} t$ but $\mathcal{E} \notin \operatorname{Lan}(\exists x P x)$. This is analogous to the case of Maehara's lemma for first-order logic with identity in [4]. 
Suppose that the last step in $\mathcal{D}$ is by $L \forall$ :

$$
\frac{A\left[\begin{array}{c}
t \\
x
\end{array}\right], \forall x A, \mathcal{E} t, \Gamma \Rightarrow C}{\forall x A, \mathcal{E} t, \Gamma \Rightarrow C} L \forall
$$

We have to consider four partitions of the conclusion:
1. $\forall x A, \mathcal{E} t, \Gamma_{1} ; \Gamma_{2} \Rightarrow C$
2. $\Gamma_{1} ; \forall x A, \mathcal{E} t, \Gamma_{2} \Rightarrow C$
3. $\mathcal{E} t, \Gamma_{1} ; \forall x A, \Gamma_{2} \Rightarrow C$
4. $\forall x A, \Gamma_{1} ; \mathcal{E} t, \Gamma_{2} \Rightarrow C$

The reader can easily see that the split-interpolants for the first two partitions are, respectively:

$$
\frac{A\left[\begin{array}{c}
t \\
x
\end{array}\right], \forall x A, \mathcal{E} t, \Gamma_{1} ; \Gamma_{2} \stackrel{I}{\Rightarrow} C}{\forall x A, \mathcal{E} t, \Gamma_{1} ; \Gamma_{2} \stackrel{I}{\Rightarrow} C} \quad \text { and } \quad \frac{\Gamma_{1} ; A\left[\begin{array}{c}
t \\
x
\end{array}\right], \forall x A, \mathcal{E} t, \Gamma_{2} \stackrel{I}{\Rightarrow} C}{\Gamma_{1} ; \forall x A, \mathcal{E} t, \Gamma_{2} \stackrel{I}{\Rightarrow} C}
$$

The third partition can be dealt with as the second partition for rule $R \exists$. In particular, by $\mathrm{IH}$ we can assume that:
(i) $\vdash \mathcal{E} t, \Gamma_{1} \Rightarrow I$
(ii) $\vdash I, A\left[\begin{array}{c}t \\ x\end{array}\right], \forall x A, \Gamma_{2} \Rightarrow C$
(iii) $\operatorname{Lan}(I) \subseteq \operatorname{Lan}\left(\mathcal{E} t, \Gamma_{1}\right) \cap \operatorname{Lan}\left(A\left[\begin{array}{c}t \\ x\end{array}\right], \forall x A, \Gamma_{2}, C\right)$

and we have to consider three mutually incompatible and exhaustive cases:

(a) $t \notin \operatorname{Ter}\left(\forall x A, \Gamma_{2}, C\right)$

(b) $t \in \operatorname{Ter}\left(\forall x A, \Gamma_{2}, C\right)$ and $t \in \mathcal{T}$

(c) $t \in \operatorname{Ter}\left(\forall x A, \Gamma_{2}, C\right)$ and $t \notin \mathcal{T}$

For each case the split-interpolant for the partition is, respectively: 


$$
\begin{gathered}
\frac{\mathcal{E} t, \Gamma_{1} ; A\left[\begin{array}{l}
t \\
x
\end{array}\right], \forall x A, \Gamma_{2} \stackrel{I}{\Rightarrow} C}{\mathcal{E} t, \Gamma_{1} ; \forall x A, \Gamma_{2} \stackrel{\exists y I\left[\begin{array}{l}
y \\
t
\end{array}\right]}{\longrightarrow} C} \quad \frac{\mathcal{E} t, \Gamma_{1} ; A\left[\begin{array}{l}
t \\
x
\end{array}\right], \forall x A, \Gamma_{2} \stackrel{I}{\Rightarrow} C}{\mathcal{E} t, \Gamma_{1} ; \forall x A, \Gamma_{2} \stackrel{I}{\Rightarrow} C} \\
\frac{\mathcal{E} t, \Gamma_{1} ; A\left[\begin{array}{l}
t \\
x
\end{array}\right], \forall x A, \Gamma_{2} \stackrel{I}{\Rightarrow} C}{\mathcal{E} t, \Gamma_{1} ; \forall x A, \Gamma_{2} \stackrel{I \wedge \mathcal{E} t}{\Longrightarrow} C}
\end{gathered}
$$

We now deal with the the fourth partition. We assume by IH that:

(i) $\vdash A\left[\begin{array}{l}t \\ x\end{array}\right], \forall x A, \Gamma_{1} \Rightarrow I$

(ii) $\vdash I, \mathcal{E} t, \Gamma_{2} \Rightarrow C$

(iii) $\operatorname{Lan}(I) \subseteq \operatorname{Lan}\left(A\left[\begin{array}{c}t \\ x\end{array}\right], \forall x A, \Gamma_{1}\right) \cap \operatorname{Lan}\left(\mathcal{E} t, \Gamma_{2}, C\right)$

We have, once again, to consider three mutually incompatible and exhaustive cases:

(a) $t \notin \operatorname{Ter}\left(\forall x A, \Gamma_{1}\right)$

(b) $t \in \operatorname{Ter}\left(\forall x A, \Gamma_{1}\right)$ and $t \in \mathcal{T}$

(c) $t \in \operatorname{Ter}\left(\forall x A, \Gamma_{1}\right)$ and $t \notin \mathcal{T}$

Mutatis mutandis, in cases (a) and (b) we reason as in the corresponding cases for the second partition of rule $R \exists$ and we find that the splitinterpolants for the partition are, respectively:

$$
\frac{A\left[\begin{array}{c}
t \\
x
\end{array}\right], \forall x A, \Gamma_{1} ; \mathcal{E} t, \Gamma_{2} \stackrel{I}{\Rightarrow} C}{\forall x A, \Gamma_{1} ; \mathcal{E} t, \Gamma_{2} \stackrel{\forall y I\left[\begin{array}{l}
y \\
t
\end{array}\right.}{\longrightarrow} C} \quad \frac{A\left[\begin{array}{l}
t \\
x
\end{array}\right], \forall x A, \Gamma_{1} ; \mathcal{E} t, \Gamma_{2} \stackrel{I}{\Rightarrow} C}{\forall x A, \Gamma_{1} ; \mathcal{E} t, \Gamma_{2} \stackrel{I}{\Rightarrow} C}
$$

sIn case (c), instead, the split-interpolant is:

$$
\frac{A\left[\begin{array}{c}
t \\
x
\end{array}\right], \forall x A, \Gamma_{1} ; \mathcal{E} t, \Gamma_{2} \stackrel{I}{\Rightarrow} C}{\forall x A, \Gamma_{1} ; \mathcal{E} t, \Gamma_{2} \stackrel{\mathcal{E} t \rightarrow I}{=} C}
$$

Indeed (iii) entails that the formula $\mathcal{E} t \rightarrow I$ satisfies the language condition since, in virtue of the assumption for case (c), we know that $t \in$ 
$\operatorname{Ter}\left(\forall x A, \Gamma_{1}\right)$ and since $\mathcal{E}$ is a logical predicate (cf. footnote 4$)$. Next the following derivations show that $\mathcal{E} t \rightarrow I$ satisfies the derivability conditions, too:

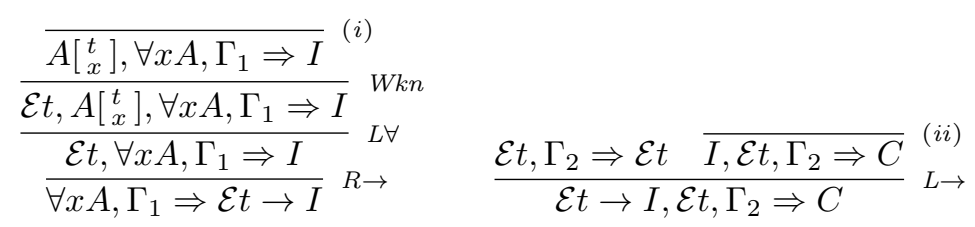

This completes the proof for $L \forall$ and we can now consider rule $R \forall$.

Suppose that the last step in $\mathcal{D}$ is by $R \forall$ :

$$
\frac{\mathcal{E} y, \Gamma \Rightarrow A\left[\begin{array}{l}
y \\
x
\end{array}\right]}{\Gamma \Rightarrow \forall x A} R \forall
$$

where $y$ is eigenvariable. As in [1] (omitting the vacuous quantifier, cf. footnote 3 ), we have to consider only one partition of the conclusion, whose split-interpolant is:

$$
\frac{\Gamma_{1} ; \mathcal{E} y, \Gamma_{2} \stackrel{I}{\Rightarrow} A\left[\begin{array}{l}
y \\
x
\end{array}\right]}{\Gamma_{1} ; \Gamma_{2} \stackrel{I}{\Rightarrow} \forall x A}
$$

The proof that $I$ satisfies the language and derivability conditions is as in [1]. Finally, we have to consider rule $E x(t)$.

Suppose the final step in $\mathcal{D}$ is by $\operatorname{Ex}(t)$ :

$$
\frac{\mathcal{E} t, \Gamma \Rightarrow C}{\Gamma \Rightarrow C} \operatorname{Ex}(t)
$$

We have only one partition to consider, namely $\Gamma_{1} ; \Gamma_{2} \Rightarrow C$. We consider two cases according to whether $t \in \operatorname{Ter}\left(\Gamma_{1}\right)$ or not and we have, respectively:

$$
\frac{\mathcal{E} t, \Gamma_{1} ; \Gamma_{2} \stackrel{I}{\Rightarrow} C}{\Gamma_{1} ; \Gamma_{2} \stackrel{I}{\Rightarrow} C} \quad \frac{\Gamma_{1} ; \mathcal{E} t, \Gamma_{2} \stackrel{I}{\Rightarrow} C}{\Gamma_{1} ; \Gamma_{2} \stackrel{I}{\Rightarrow} C}
$$


The fact that $I$ satisfies the derivability conditions is obvious in both cases: we just have to apply $\mathrm{IH}$ and then an instance of rule $E x(t)$. It is also easy to see that $I$ satisfies the language condition too: in the first case $I$ satisfies the language condition because $(\mathcal{E} \notin \operatorname{Lan}(I)$ and $)$ if $t \in \operatorname{Ter}(I)$ then, by IH, it must be in $\operatorname{Ter}\left(\Gamma_{2}, C\right)$ and we are assuming that it is in $\operatorname{Ter}\left(\Gamma_{1}\right)$; but so does in the second case, since here we are assuming that $t \notin \operatorname{Ter}\left(\Gamma_{1}\right)$ and hence $t \notin \operatorname{Ter}(I)$.

From Maehara's lemma for G3ie ${ }^{\mathcal{T}}$, it is immediate to prove Craig's interpolation theorem.

Theorem 11 (Craig's interpolation for $\mathrm{G}^{2} \mathrm{ie}^{\mathcal{T}}$ ). If $A \Rightarrow B$ is derivable in $\mathrm{G} \mathrm{Ti}^{\mathcal{T}}$ then there exists a formula $I$ such that $\vdash A \Rightarrow I$ and $\vdash I \Rightarrow B$ and $\operatorname{Lan}(I) \subseteq \operatorname{Lan}(A) \cap \operatorname{Lan}(B)$.

Proof: Let $A \Rightarrow B$ be derivable in $\mathrm{G} \mathrm{ie}^{\mathcal{T}}$ and let us consider the partition $A ; \varnothing \Rightarrow B$ of $A \Rightarrow B$. By Lemma 10, this partition has a split-interpolant, namely there exists a $I$ such that $A ; \varnothing \stackrel{I}{\Rightarrow} B$. Hence $\vdash A \Rightarrow I$ and $\vdash I \Rightarrow B$ and $\operatorname{Lan}(I) \subseteq \operatorname{Lan}(A) \cap \operatorname{Lan}(B)$ by Definition 8 .

Comparing our proof of interpolation with that of [1], it appears that ours is direct in the sense that it relies exclusively on the rules of $\mathrm{G} \mathrm{ie}^{\mathcal{T}}$, with no need to go through an equivalent system.

\section{Interpolation for the implication-free fragment}

In this section we consider interpolation for sequents in the fragment of the language without implication $($ nor $\perp$ ). First, we show that for the procedure given in Lemma 10 (henceforth Proc) there exists a bound on the number of implications introduced in the interpolants. Next, we consider the class of implication-free derivable partitions $\Pi_{1} ; \Pi_{2} \Rightarrow F$ such that $\Pi_{2}$ contains no existential atom. We show that for sequents in this class, Proc can be modified in such a way that it outputs implication-free interpolants. Notice that the assumption that no existence atom occurs in the second component of the end-sequent is not restrictive as long as one aims to prove interpolation for sequents representing theorems of ILE, since their second component is empty (cf. Theorem 11).

We say that a sequent calculus $\mathrm{G}$ interpolates for a fragment $\mathcal{F}$ of the language $\mathcal{L}^{\prime}$ if whenever $\vdash \Gamma_{1} ; \Gamma_{2} \stackrel{I}{\Rightarrow} C$ in $\mathrm{G}$ with $\Gamma_{1}, \Gamma_{2}, C \in \mathcal{F}$, we 
have that $I \in \mathcal{F}$. It is well-known that LJ interpolates for the $\{\wedge, \vee, \exists, \forall\}$ fragment. Nevertheless, in $[1, \S 5.1]$ it is conjectured LJE and $\operatorname{LJE}\left(\Sigma_{\mathcal{L}}\right)$ do not interpolate for the $\{\wedge, \vee, \exists, \forall\}$-fragment because rule $L \forall$ does not interpolate for this fragment. The problem, roughly, is that the procedure given in $[1$, Theorem 4] might introduce an implication in the interpolant of the conclusion of an instance of $L \forall$.

We are now going to show that the calculi G3ie and $\mathrm{G}_{3} \mathrm{ie}^{\mathcal{T}}$ are better behaved with respect to interpolation for the $\{\wedge, \vee, \exists, \forall\}$-fragment in that: Proposition 12. If $\Gamma_{1} ; \Gamma_{2} \Rightarrow C$ is a $\mathrm{G} \mathrm{ie}^{(\mathcal{T})}$-derivable sequent in the $\{\wedge, \vee, \exists, \forall\}$-fragment and we apply Proc to its derivation, we obtain an interpolant I such that:

( $\alpha$ ) If an implication occurrs in $I$, its antecedent is of the form $\mathcal{E} t ;^{5}$

$(\beta)$ If $\#_{\circ}(\Gamma)$ stands for the number of occurrences of the symbol $\circ$ in $\Gamma$, then

$$
\#_{\rightarrow}(I) \leq\left[\#_{\forall}\left(\Gamma_{1}\right)\right] \times\left[\#_{\mathcal{E}}\left(\Gamma_{2}\right)+\#_{\forall}(C)+\#_{\exists}\left(\Gamma_{2}\right)\right]
$$

Proof: First of all, by inspecting Proc we immediately see that an implication may occur in the interpolant of an implication-free sequent only when in its derivation there is an instance of $L \forall$ whose conclusion is an instance of subcase (c) of the fourth partition considered in Lemma 10, i.e., it is of the form

$$
(\dagger) \frac{A\left[\begin{array}{l}
t \\
x
\end{array}\right], \forall x A, \Delta_{1} ; \mathcal{E} t, \Delta_{2} \Rightarrow D}{\forall x A, \Delta_{1} ; \mathcal{E} t, \Delta_{2} \Rightarrow D} L \forall \quad(\ddagger) \text { with } t \notin \mathcal{T} \text { and } t \in \operatorname{Ter}\left(\forall x A, \Delta_{1}\right)
$$

Let us call quasi-implicative an arbitrary instance of the fourth partition of $L \forall$ and fully-implicative one that falls under case (c). Thus, an implication may occur in the interpolant of an implication-free conclusion of an instance of a rule of $\mathrm{G} \mathrm{ie}^{\mathcal{T}}$ if and only if it is a fully-implicative instance of $L \forall$.

The claim $(\alpha)$ holds since the interpolant of a conclusion of a fullyimplicative instance of $L \forall$ is $\mathcal{E} t \rightarrow J$ (where $J$ is the interpolant of the premiss).

To prove claim $(\beta)$ we analyze the derivation of $\Gamma_{1} ; \Gamma_{2} \Rightarrow C$ bottomup, as is normally done in proof-search procedures. The first thing to notice is that no case of Proc for the $\{\wedge, \vee, \exists, \forall\}$-fragment switches the position of

${ }^{5}$ And not a formula of arbitrary complexity as in [1, Thm. 4] 
a (sub)formula occurring in a rule instance: in moving from the conclusion to the premiss(es) nothing goes from one component of the antecedent (or from one side of the sequent) to the other. As a consequence we have that $\Gamma_{1} ; \Gamma_{2} \Rightarrow C$ has been concluded by a quasi-implicative instance of $L \forall$ only if:

1. its principal formula $\forall x A$ is a subformula of (some formula in) $\Gamma_{1}$, and

2. its principal formula $\mathcal{E} t$

(a) is a subformula of $\Gamma_{2}$, or

(b) it has been introduced (bottom-up) by an instance of $E x(t)$, or

(c) it has been introduced (bottom-up) by an instance either of $R \forall$ whose principal formula is a subformula of $C$ or of $L \exists$ whose principal formula is a subformula of $\Gamma_{2}$.

We immediately have that the number of (quasi- and) fully-implicative instances of $L \forall$ is bounded by a function of the number of universal quantifiers occurring in $\Gamma_{1}$, namely $\# \rightarrow(I) \leq\left[\#_{\forall}\left(\Gamma_{1}\right)\right] \times m$, for some $m$.

Now we show that $m=\#_{\mathcal{E}}\left(\Gamma_{2}\right)+\#_{\forall}(C)+\#_{\exists}\left(\Gamma_{2}\right)$. This will done by identifying when the quasi-implicative instance of $L \forall$ in $(\dagger)$ is fullyimplicative, i.e. it satisfies $(\ddagger)$. If its principal formula $\mathcal{E} t$ is a subformula of $\Gamma_{2}$, it is fully-implicative if and only if $t \notin \mathcal{T}$ and $t \in \forall x A, \Delta_{1}$. Hence $\# \mathcal{E}\left(\Gamma_{2}\right)$ goes into $m$. If, instead, its principal formula $\mathcal{E} t$ has been introduced by a lower instance of $E x(t)$, it is never fully-implicative since $t \in \mathcal{T}$. Hence nothing goes into $m$. Lastly, if its principal formula $\mathcal{E} t$ has been introduced by a lower instance of one of $R \forall$ and $L \exists$, say

$$
\frac{\Sigma_{1} ; \mathcal{E} t, \Sigma_{2} \Rightarrow B\left[\begin{array}{l}
t \\
z
\end{array}\right]}{\Sigma_{1} ; \Sigma_{2} \Rightarrow \forall z B} R \forall
$$

by the variable condition on $R \forall$ we immediately get that $t$ is a variable (hence we know $t \notin \mathcal{T}$ ) that does not occur in $\Sigma_{1}$. Therefore, $t$ can be in $\operatorname{Ter}\left(\forall x A, \Delta_{1}\right)$ only if it has been introduced (bottom-up) by some rule instance occurring between $R \forall$ and $(\dagger)$. The only rule that can introduce new (free) occurrences of $t$ in the first component of its premiss is $L \forall$. Hence the first quasi-implicative instance of $L \forall$ (with $\mathcal{E} t$ principal) cannot be fully-implicative (the only occurrence of $t$ in its antecedent is the one in $\mathcal{E} t$ ) and each other one is fully-implicative (provided the quantification in the principal formula of the first one wasn't vacuous). Hence $\#_{\forall}(C)$ and $\#_{\exists}\left(\Gamma_{2}\right)$ goes into $m$. 
Now we give an alternative procedure showing that, under a plausible assumption, G3ie ${ }^{\mathcal{T}}$ interpolates for the $\{\wedge, \vee, \exists, \forall\}$-fragment.

Proposition 13. The calculus $\mathrm{G} \mathrm{e}^{(\mathcal{T})}$ interpolates for the $\{\wedge, \vee, \exists, \forall\}$ fragment provided that we exclude end-sequents with existence atoms occurring in their second component.

Proof: Suppose we are applying Proc to the derivation of a sequent $\Pi_{1} ; \Pi_{2} \Rightarrow F$ satisfying the hypothesis of the Proposition 13. By Proposition 12 , we know that an instance of $L \forall$ is fully-implicative only if its principal formulas $\mathcal{E} y$ has been introduced in the second component by a lower instance of either $L \exists$ or $R \forall$, and $y$ has been introduced in its firstcomponent by another (in-between) instance of $L \forall$. In particular, the first instance of $L \forall$, i.e. the one introducing free occurrences of $y$ in the first component, is a (non-fully-implicative) instance of case (a) of the fourth partition for $L \forall$ in Lemma 10.

Let us consider a procedure Proc* that is like Proc save that in case (a) of the fourth partition for $L \forall$ it moves the existence atom $\mathcal{E} t$ from the second component of its conclusion to the first component of its premiss:

$$
\frac{\mathcal{E} t, A\left[\begin{array}{l}
t \\
x
\end{array}\right], \forall x A, \Delta_{1} ; \Delta_{2} \stackrel{I}{\Rightarrow} D}{\forall x A, \Delta_{1} ; \mathcal{E} t, \Delta_{2} \stackrel{\forall y I\left[\begin{array}{l}
y \\
t
\end{array}\right.}{\longrightarrow} D} L \forall, \quad t \notin \operatorname{Ter}\left(\forall x A, \Delta_{1}\right)
$$

Maehara's lemma holds for Proc*: we have just moved the existence atom $\mathcal{E} t$ from one component to the other. This difference has no impact for the language condition since $\mathcal{E}$ is a logical predicate and $t \notin \operatorname{Ter}\left(\forall y I\left[\begin{array}{c}y \\ t\end{array}\right]\right)$. As for the derivability conditions, the only difference is that now we have to introduce $\mathcal{E} t$ via an instance of weakening in the derivation of $\forall y I\left[\begin{array}{l}y \\ t\end{array}\right], \mathcal{E} t, \Delta_{2} \Rightarrow$ $C$ instead of introducing it via weakening in the one of $\forall x A, \Delta_{1} \Rightarrow \forall y I\left[\begin{array}{l}y \\ t\end{array}\right]$ (as in lemma 10).

Let us consider an arbitrary quasi-implicative instance of $L \forall$ occurring in the derivation of $\Pi_{1} ; \Pi_{2} \Rightarrow F$ with principal formula $\mathcal{E} y$ (introduced by a lower instance of one of $R \forall$ and $L \exists$ ). We can easily show that it is not a fully-implicative instance since it must fall under the modified case (a) above. To witness, we have already shown that the first quasiimplicative instance of $L \forall$ occurring above the introduction of $\mathcal{E} y$ falls under case (a) and, given that Proc* moves $\mathcal{E} y$ to the first component of 
the premiss of this rule instance and no rule instance can move it back to the second component nor introduce another instance of $\mathcal{E} y$ (since we are considering derivations satisfying the pure-variable convention), no other quasi-implicative instance of $L \forall$ with principal formula $\mathcal{E} y$ can occur above the first one. Thus we never apply a fully-implicative instance of $L \forall$ under Proc*, and this is enough to prove the proposition.

From the perspective of the numeric bound given for Proc in Proposition 12 , we now have that $\#_{\mathcal{E}}\left(\Gamma_{2}\right)=0$ by hypothesis of the proposition and that Proc* is defined so that $\#_{\forall}(C)+\#_{\exists}\left(\Gamma_{2}\right)$ is replaced by 0 . Hence $\# \rightarrow(I)=0$.

\section{Conclusion}

In this paper we presented an improvement on the previous work by Baaz and Iemhoff on cut elimination and interpolation for ILE. In particular, we have shown that ILE admits a fully cut-free systematization in sequent calculus, which allows a direct constructive proof of interpolation, and we have shown that if an implication occurs in the interpolant of an implication-free sequent, then its antecedent must be an atom of existence. Moreover, we have also shown that (under a plausible assumption) our cut-free calculi interpolate for the $\{\wedge, \vee, \exists, \forall\}$-fragment.

This paper is also an improvement on the previous work on interpolation in first-order theories, especially [4]. In [4] it is shown how to extend interpolation from classical and intuitionistic logic to singular geometric theories, a subclass of geometric theories investigated in [6]. Interestingly, singular geometric theories are subjected to the condition that individual constants do not occur in any axiom. ILE is clearly an example of a theory outside the singular geometric class, since individual constants occur necessarily in existential axioms. Therefore, $\mathrm{G} \mathrm{ie}^{\mathcal{T}}$ is a calculus not falling within the singular geometric class for which interpolation holds. This motivates further interest in generalizing the approach of [4] and we leave the task to future work. 


\section{References}

[1] M. Baaz and R. Iemhoff. On interpolation in existence logics, Logic for Programming, Artificial Intelligence, and Reasoning ed. by G. Sutcliffe and A. Voronkov, vol. 3835 of Lecture Notes in Computer Science. Springer, 2005, pp. 697-711.

[2] M. Baaz and R. Iemhoff, Gentzen calculi for the existence predicate, Studia Logica, vol. 82, no. 1 (2006), pp. 7-23.

[3] M. Beeson, Foundations of Constructive Mathematics. Springer, 1985.

[4] G. Gherardi, P. Maffezioli, and E. Orlandelli, Interpolation in extensions of first-order logic, Studia Logica (2019), pp. 1-30. (published online).

[5] S. Maehara, On the interpolation theorem of Craig. Suugaku, vol. 12 (1960), pp. 235-237. (in Japanese).

[6] S. Negri, Contraction-free sequent calculi for geometric theories with an application to Barr's theorem, Archive for Mathematical Logic, vol. 42, no. 4 (2003), pp. 389-401.

[7] S. Negri, Proof analysis in modal logic, Journal of Philosophical Logic, vol. 34, no. (5-6) (2005), pp. 507-544.

[8] S. Negri and J. von Plato, Structural Proof Theory. Cambridge University Press, 2001.

[9] D. Scott, Identity and existence in intuitionistic logic, [in:] M. Fourman, C. Mulvey, and D. Scott, editors, Application of Shaves. Springer, 1979, pp. $660-696$.

[10] A. S. Troelstra and H. Schwichtenberg, Basic Proof Theory. Cambridge University Press, 2nd edition, 2000.

Departamet de Filosofia

Universitat de Barcelona

Montalegre, 6, 08001 Barcelona, Spain

e-mail: paolo.maffezioli@ub.edu

Dipartimento di Filosofia e Comunicazione

Università di Bologna

Via Azzo Gardino, 23, 40122, Bologna, Italy

e-mail: eugenio.orlandelli@unibo.it 